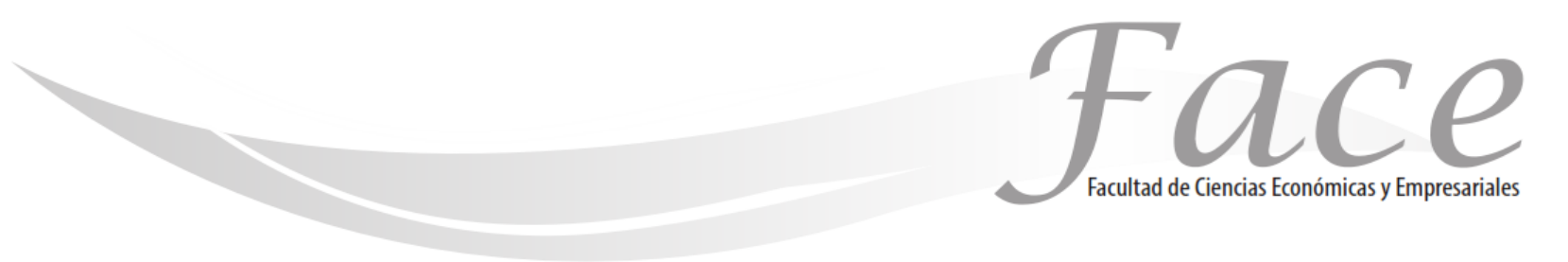

ISSN Impreso: 1794-9920

ISSN Electrónico: 2500-9338

Volumen $20-\mathrm{N}^{\circ} 2$

Año 2020

Págs. $30-44$

(c)

\title{
POBREZA LABORAL DEL SIGLO XXI EN MÉXICO: ANÁLISIS DESDE LA PERSPECTIVA DE GÉNERO *
}

\author{
Mariana Sánchez Licona * \\ Enlace ORCID: https://orcid.org/0000-0003-4308-848X \\ Eduardo Rodríguez Juárez ** \\ Enlace ORCID: https://orcid.org/0000-0002-2232-7544
}

Fecha de Recepción: Octubre 2 de 2020

Fecha de Aprobación: Diciembre 20 de 2020

Resumen:

El objetivo de este trabajo fue analizar las aproximaciones teóricas para explicar la pobreza como un fenómeno esencialmente distributivo. Se revisan algunas de las explicaciones que propone la literatura empírica que estudia la pobreza vista desde la perspectiva de género. Se encuentra que la pobreza es derivada de un problema esencialmente distributivo que embiste principalmente a las mujeres mexicanas.

Palabras clave: Pobreza, Distribución, Empleo, Mujeres, Género.

* El presente artículo es uno de los resultados de investigación obtenidos para sustentar una tesis, titulada "Distribución, pobreza y trabajo de las mujeres: Análisis teórico y empírico para el caso mexicano", la cual presentó la autora con base en la orientación de su coautor como requisito para obtener el grado de Maestra en Estudios Económicos. Instituto de Ciencias Económico Administrativas, México. Universidad Autónoma del Estado de Hidalgo, México.

${ }^{* *}$ Maestra en Estudios Económicos, Profesora por asignatura del Área Académica de Economía del Instituto de Ciencias Económico Administrativas, México. Universidad Autónoma del Estado de Hidalgo, México. Contacto: msanchez licona@uaeh.edu.mx

*** Doctor en Ciencias Económicas. Profesor Investigador del área Académica de Economía del Instituto de Ciencias Económico Administrativas, México. Universidad Autónoma del Estado de Hidalgo, México Contacto: eduardor@uaeh.edu.mx 



\title{
LABOR POVERTY IN THE XXI CENTURY IN MEXICO: ANALYSIS FROM A GENDER PERSPECTIVE
}

\begin{abstract}
:
The objective of this work was to analyze the theoretical approaches to explain poverty as an essentially distributive phenomenon. Some of the explanations proposed by the empirical literature that studies poverty seen from a gender perspective are reviewed. It is found that poverty is derived from an essentially distributive problem that affects mainly Mexican women.
\end{abstract}

Keywords: Poverty, distribution, employement, women, gender.

\section{POBREZA DO TRABALHO NO SÉCULO XXI NO MÉXICO: ANÁLISE DA PERSPECTIVA DE GÊNERO}

\section{Resumo:}

O objetivo deste trabalho foi analisar as abordagens teóricas para explicar a pobreza como um fenômeno essencialmente distributivo. São revisadas algumas das explicações propostas pela literatura empírica que estuda a pobreza desde uma perspectiva de gênero. Verificou-se que a pobreza deriva de um problema essencialmente distributivo que afeta principalmente as mulheres mexicanas.

Palavras-chave: Pobreza, distribuição, emprego, mulheres, gênero. 


\section{INTRODUCCIÓN:}

La búsqueda de alternativas para superar la pobreza es una de las preocupaciones de los diseñadores de política pública. A pesar de la gravedad del problema, pocos son los estudios recientes en donde se aborda, desde una perspectiva económica las causas que pueden originarla, los impactos y consecuencias que produce $y$, por supuesto, las posibles vías para superarla. La presente investigación estudia no solamente la pobreza, sino además el hecho de que exista una mayor cantidad de mujeres que de hombres en condiciones de pobreza, es decir, el fenómeno de la feminización de la pobreza en México y en el contexto internacional. De esta forma, el objetivo del presente documento es analizar las aproximaciones teóricas para explicar la pobreza como un fenómeno esencialmente distributivo. Además, se revisan algunas de las explicaciones que propone la literatura empírica que estudia la pobreza vista desde la perspectiva de género.

Tal y como lo reconoce la Organización de las Naciones Unidas (ONU), es de gran importancia buscar caminos hacia la erradicación de la pobreza, por tanto, no es extraño que el primero de los Objetivos de Desarrollo Sostenible trazados por la propia ONU en la Agenda 2030, sea justamente erradicar la pobreza en todas sus formas (Organización de las Naciones Unidas, 2018). A pesar de que se ha avanzado en el terreno de su medición y de que las medidas para erradicarla están en constante evolución y rediseño, la pobreza sigue siendo un fenómeno que lacera a nuestras sociedades.

En concordancia, el quinto y no menos importante de los objetivos es lo referente a la promoción de la igualdad de género y el empoderamiento de las mujeres y las niñas, puesto que, aún en el siglo XXI se siguen presentando inequidades que atentan contra los derechos y la dignidad de las mujeres en distintos aspectos. Como bien lo observa el trabajo de Ordoñez (2018), la discriminación, por diversos motivos, que existe en México suele afectar más en los grupos vulnerables, tales como la niñez, los adultos mayores, las mujeres y los pueblos originarios. El estudio muestra que la discriminación parece aún más agresiva para el caso de las mujeres pobres mexicanas.

Si bien se han realizado trabajos que explican las causas tanto de que exista una alta tasa de mujeres en condiciones de pobreza (y pobreza extrema), como de que las condiciones de esa pobreza se agudicen en el caso de las mujeres, el panorama que se tiene de lo que ello ha implicado para el caso mexicano no es completo. En este sentido, el problema radica en la necesidad de explicar teórica y empíricamente la pobreza laboral por género para el caso mexicano en la época más reciente, es decir, a partir de inicios del siglo XXI, puesto que, como se mencionó, las implicaciones del problema de la pobreza son distintas para hombres y para mujeres, y el paradigma para estudiar la pobreza comparando condiciones entre los mismos es el mercado laboral. Es importante mencionar que, como lo señalan algunos autores, las problemáticas que tienen que ver con la incorporación de la mujer al aparato productivo, pueden observarse en forma clara a partir de los años posteriores a la década de 1990, ya que es posterior al cambio en el estilo de las políticas públicas cuando puede notarse una diferencia esencial en la forma en que las mujeres se adhieren al mercado laboral y las condiciones bajo las cuales lo hacen.

El presente trabajo cobra relevancia al voltear la mirada hacia algunos de los temas que lastiman a la sociedad mexicana la pobreza que enfrenta casi el $44 \%$ de la población en México (Consejo Nacional de Evaluación de la Política de Desarrollo Social, 2016), abordándolo desde la perspectiva de las brechas entre hombres y mujeres, desde la perspectiva de género, con lo cual se da luz hacia la economía de las mujeres, uno de los sectores que, si bien ha sido estudiado con mayor frecuencia en los últimos años, aún sigue presentándose como un grupo vulnerable y con problemas serios de discriminación. 
El presente documento muestra un panorama estadístico general para el caso mexicano: el primer apartado realiza una construcción teórica sobre el problema de investigación: ¿es la pobreza un problema distributivo? muestra las diferentes visiones teóricas que visualizan la respuesta a la pregunta anterior, así como el debate entre ellas a partir de la literatura empírica analizada. En el segundo apartado se realiza una revisión a la evidencia empírica para mostrar la realidad de las mujeres mexicanas en condición de pobreza laboral. Finalmente, se muestran algunas conclusiones y reflexiones a las cuales ha dado paso la investigación.

\section{INTRODUCCIÓN:}

\subsection{PROBLEMA DISTRIBUTIVO EN LA TEORÍA ECONÓMICA}

El punto central de este apartado es generar un debate entre las ideas de la escuela tradicional del pensamiento económico y las distintas críticas que se han conformado alrededor de ella. En estricto sentido, lo que se busca es contrastar las diferentes concepciones de la pobreza: si tiene que ver con una asignación asimétrica del ingreso, el problema es claramente económico. Si, por el contrario, la pobreza tiene origen en cuestiones ajenas al funcionamiento de los mercados, el problema se tendría que tratar desde la esfera de lo social. La idea que defiende este trabajo de investigación es que existe una distribución asimétrica del ingreso que provoca el empobrecimiento de los agentes económicos que se encuentran en desventaja $\mathrm{y}$, dentro de esa distribución asimétrica propiciada por los mecanismos de mercado, existe otra asimetría en la distribución del ingreso.

${ }^{1}$ Como lo explica Klimovsky (2000), hay dos cosas importantes a resaltar en la teoría neoclásica de los precios: por un lado, la determinación de los precios de equilibrio como resultado matemático de un sistema de

\section{MARCO TEÓRICO}

Teoría Económica Ortodoxa

Uno de los principios del marginalismo más arraigados en el aprendizaje de la ciencia económica es precisamente el relacionado a la desvinculación de la política con la economía (Klimovsky, 2000): para que la armonización de las decisiones económicas e intereses de los agentes pueda ocurrir, el Estado y cualquier otra fuerza que pudiera intervenir en el proceso de mercado, debe mantenerse al margen de la economía. Para la teoría neoclásica, la distribución del ingreso y la pobreza quedan fuera del análisis de la dinámica de mercados, dado que estos (en condiciones de competencia perfecta) logran asignaciones eficientes. El marco analítico bajo el cual la teoría neoclásica explica sus postulados es conocido como Modelo de Equilibrio General, y se basa en ciertos supuestos importantes para el desarrollo de este.

En primer lugar, el MEG exige condiciones de competencia perfecta ${ }^{1}$, lo cual significa que se habla de una economía donde es el mercado la única institución capaz de asignar precios en forma eficiente. Además, implica que la entrada y salida de empresas del mercado es completamente libre, o bien, que existe un número suficientemente grande de oferentes y demandantes, de tal manera que ninguno por sí solo (o en grupos) pueda influir en la determinación de los precios. En suma, el precio es un resultado de la interacción entre oferentes y demandantes dentro del mercado. Dado que los precios y las asignaciones son los hallazgos del equilibrio, tanto oferentes como demandantes tienen la misma importancia dentro del proceso de mercado. Las acciones de todos los agentes son igualmente importantes para la acción del mercado, y únicamente son diferenciables por gustos, preferencias y dotaciones iniciales. Esto se refiere a la existencia de una sociedad simétrica, sin clases

ecuaciones y, por otro lado, la conformación de estos mediante la libre competencia. 
sociales, sin una división marcada por situaciones económicas entre empresarios y trabajadores ${ }^{2}$.

Es muy importante enfatizar en esta idea: partir del supuesto de que existen sociedades simétricas implica que en el análisis no cabe la posibilidad de que existan clases sociales (como sí la hay en la teoría clásica, y más específicamente, en la marxista) ${ }^{3}$. En otras palabras, la teoría neoclásica rechaza la idea de que el conflicto distributivo exista dentro de la esfera económica. Tomando este principio como supuesto básico, la conclusión a la que arriba el marginalismo es evidente, y sienta las bases de una corriente de pensamiento que no considera a la desigualdad y la pobreza como patologías que haya que corregir desde la propia economía, sino desde cualquier otra área. La teoría neoclásica muestra que, al ser la ganancia un resultado de factores que estrictamente tienen que ver con la tecnología disponible, difícilmente se relaciona con un acuerdo asimétrico o injusto para una de las partes que conforman el mercado. Es decir, la desigualdad no puede concebirse como un problema de carácter distributivo, porque las ganancias son originadas no por factores económicos, sino tecnológicos. Entonces, no están en conflicto trabajadores y empresarios; no hay problema de distribución porque el mercado asigna de manera eficiente, ya que el trabajador es remunerado conforme a su productividad.

\section{Análisis de la pobreza en el contexto neoclásico}

En el contexto de la economía neoclásica se han desarrollado trabajos que sugieren que la pobreza debe abordarse como un problema social: puesto que los mercados realizan asignaciones eficientes, la persona pobre lo es porque llega al mercado a competir bajo condiciones desiguales. En otras palabras, porque no tiene todas las capacidades para desarrollarse a plenitud y dejar de ser pobre. Una de

\footnotetext{
2 "Los servicios de los factores no se diferencian de la generalidad de los bienes en cuanto a la determinación de sus precios. (...) no existe una diferencia substancial entre el servicio de un capital y el de un tipo particular de trabajo o de tierra. En este contexto, no hay lugar para las clases sociales." (Klimovsky, 2000, p. 84-85).

3 Para los neoclásicos, el conflicto distributivo existe cuando los mercados no se dejan en libertad. Es decir, cuando existe competencia imperfecta, o
}

las visiones sobre pobreza más frecuente retomadas en los estudios económicos y sociales que toman a la pobreza como problema de investigación es la que ha desarrollado el Premio Nobel de Economía 1998, Amartya K. Sen, cuyos trabajos refieren a la pobreza desde el enfoque de capacidades, como se le ha llamado.

Según Sen (1981), los pobres son aquellos que tienen niveles de consumo por debajo de ciertas normas establecidas como mínimas, o bien, tienen niveles de ingreso por debajo de lo que Sen (1981) define como línea de pobreza. Para efectos de una correcta medición de la pobreza, considera que también hay que especificar muy claramente al grupo cuyos intereses deben atenderse implícitamente al atender la pobreza. Amartya Sen señala que el foco del concepto de pobreza habrán de ser los pobres como tal y su bienestar, sin importar cuáles sean los factores que estén afectando. Así, se llega a una conclusión muy importante con respecto al pensamiento de Amartya Sen: Las causas y consecuencias de la pobreza constituyen en sí mismas un tema de estudio muy amplio que puede separarse considerablemente del problema de la conceptualización de la pobreza. La filosofía de Amartya Sen es muy clara en definir a la pobreza como un problema considerablemente distinto al de la desigualdad. Es decir, la pobreza no es un tema de distribución del ingreso, puesto que tienen naturalezas distintas.

\section{Crítica a la Economía Ortodoxa}

Existen otras visiones, en las cuales se ha dejado de manifiesto que el problema puede ser económico. Por ejemplo, en el contexto internacional, estudios como los presentados por Stiglitz (2012) y Piketty (2014) notan que, a pesar de la insistencia neoclásica porque los salarios sean justos en la medida en que retribuyen la productividad marginal del trabajo ${ }^{4}$, el problema de

\footnotetext{
bien cuando el Estado interviene, es cuando existen ineficiencias en las asignaciones, lo cual no permite que el proceso distributivo se realice en forma adecuada. Así, la desigualdad existe, pero es un fenómeno completamente ajeno a un mercado de competencia perfecta. Este análisis se profundiza en textos como el de Velázquez, Rodríguez y González (2017).

4 El salario es justo, pues se retribuye al trabajador según su propia aportación a la producción (Velázquez, Rodríguez, \& González, 2017).
} 
la distribución sigue presente. En el caso de Stiglitz (2012), estima que sólo una pequeña parte de la población mundial (el 1\%) tiene en posesión el ingreso equivalente a lo que requieren para vivir dignamente el $99 \%$ restante: el trabajo es una evidencia interesante para analizar el problema de la distribución mundial del ingreso, así como los niveles de pobreza a nivel internacional. Por su parte, la tesis planteada por Piketty (2014) resulta de igual forma interesante, puesto que concluye que existen niveles altos de concentración del ingreso dado que históricamente las tasas de rendimiento del capital han sido considerablemente mayores a las tasas de crecimiento económico de los países que analiza (Francia, Reino Unido y EUA). Del texto de Piketty (2014) puede inferirse que la concentración del ingreso refiere a una división en clases sociales, es decir, que la sociedad no es necesariamente simétrica, pues existe un cierto antagonismo entre empresariado y trabajadores, que culmina finalmente en una apropiación de ingreso nacional por parte del capitalista, superior (por mucho) a la que realiza el trabajador.

Para el caso mexicano, existen estudios como el presentado por Esquivel (2015), en donde realiza un análisis del reparto capital-trabajo: mediante un estudio de la distribución del producto entre los factores de la producción desde 1970 y hasta 2012, el informe muestra que, en todos los años, la concentración de ingreso por ganancias (parte que corresponde al empresariado) es muy superior a los ingresos que corresponden a la clase trabajadora. Incluso, puede notarse que, a partir de la década de los años noventa, existe una tendencia a crecer de la concentración por el factor capital, mientras que es notable una disminución en la concentración de ingresos por el factor trabajo. En correspondencia con los análisis de Piketty (2014), Esquivel (2015) encuentra una desigualdad en ingresos entre trabajadores y capitalistas que sigue vigente para el caso mexicano y que evidencia que (contrario a la afirmación neoclásica) la distribución puede no ser un fenómeno estrictamente social o tecnológico, sino de naturaleza económica y que debe atenderse como tal.
Los economistas clásicos fueron los primeros en plantear el conflicto distributivo ellos consideran a las remuneraciones de los factores de la producción como variables determinantes de la distribución del ingreso. Como consecuencia de ello, habrá un sector poblacional que perciba mayor ingreso que otro, generando asimetrías en la distribución y, por tanto, pobreza en determinados grupos de la población. Es el caso de Smith (1776), que considera que existen esencialmente dos variables distributivas constituidas por las retribuciones de los factores productivos trabajo y capital. Estas variables distributivas se encuentran en una constante contradicción, puesto que, si los salarios son altos, los beneficios son menores. Es decir, existen dos agentes representativos en la economía: por un lado, se encuentra el trabajador y por otro lado el capitalista. Las diferencias entre ellos radican en la posesión de los medios de producción, pues mientras que el trabajador sólo es dueño de su fuerza de trabajo, el capitalista es dueño de los medios de producción. La asimetría que esto genera radica en que, si el trabajador decide dejar de trabajar, sencillamente no tendrá acceso a los satisfactores de necesidades más elementales. Por su parte, si el capitalista decide no producir o no hacer nada con su capital, puede vender sus medios de producción 0 bien, vivir de su propio capital sin mayores complicaciones.

En este sentido, a pesar de que Smith manifiesta que el salario es un acuerdo entre capitalista y trabajador, también es cierto que el acuerdo es asimétrico, pues necesariamente alguien está ganando en el intercambio. Es decir, dado que en la negociación el capitalista se encuentra en ventaja sobre el trabajador (porque tiene en su poder los medios de producción), el arreglo favorece siempre al capitalista, quien busca obtener las mayores ganancias posibles, generándose con ello asimetrías en la distribución del ingreso y, por lo tanto, un empobrecimiento crónico de la clase trabajadora. La forma que sugiere Smith para lograr el ajuste de dichas asimetrías distributivas es asegurar un salario humanitario o de subsistencia, el cual debe ser mínimamente suficiente para que el trabajador asegure su supervivencia y la de su familia. La 
referencia de Smith también es interesante en el sentido de que plantea que el trabajo del hombre debe generar los ingresos suficientes para satisfacer las necesidades de la familia.

A pesar de que considera que también la mujer aporta en términos de trabajo (además de ser la responsable de la crianza de los hijos) para el ingreso familiar, señala que el ingreso de ella es considerablemente menor al ingreso del esposo. Si bien Smith no profundiza en el análisis de esta idea, sí se sugiere que la distribución del ingreso no solamente es asimétrica entre clase capitalista y clase trabajadora, sino que también es asimétrica dentro de la clase que posee menor proporción del ingreso (trabajadores). Es decir, a pesar de que Adam Smith no analiza las diferencias en ingresos por género, aporta el indicio de la teoría clásica para reconocer que la distribución del ingreso no solamente está concentrada en la clase capitalista, sino que también se concentra dentro de la propia clase trabajadora hacia los hombres.

Por su parte, Ricardo (1817) también observa que el salario y los beneficios (además de la renta de la tierra) constituyen variables distributivas del ingreso. Sin embargo, mientras que Smith señala que el crecimiento de la población (sobre todo, de la población en condiciones de pobreza) favorecería al crecimiento de la producción y, por lo tanto, a la riqueza de las naciones, Ricardo sostiene que la elevación en los salarios no es benéfica, puesto que disminuye las ganancias del capital, pero, además porque promueve el acelerado crecimiento poblacional, derivando nuevamente en desempleo y disminución de ganancias capitalistas.

La postura marxista (1867), señala que la acumulación del capital tiene como origen la apropiación que realiza el capitalista de un tiempo de trabajo que no paga al trabajador (plusvalía). De esta forma, sin modificar la cantidad de capital que se ponga a trabajar, el capitalista reduce sus costos salariales (parte variable de su capital) para mantener constante su capital. Marx igualmente encuentra la contraposición entre los intereses del capitalista y los del trabajador y, dado que señala a las ganancias y los salarios como variables distributivas, esta contraposición, aunado a la posesión de los medios de producción, son lo que genera las asimetrías en la distribución del ingreso. Es interesante observar que, a pesar de que existen coincidencias importantes con otros economistas clásicos, también hay diferencias que han hecho que la teoría marxista en nuestros días se constituya como todo un campo de estudio dentro de la economía. Una diferencia central, por ejemplo, con Smith y Ricardo, es que Marx no es partidario del libre mercado. La propuesta de Marx hacia la solución de las asimetrías generadas por el mercado en la distribución del ingreso tiene que ver con la administración y operación de los medios de producción por parte del Estado.

Análisis de la pobreza laboral por género desde la crítica

Aunque en la actualidad hay un trabajo relativamente joven que trata el tema de la pobreza vista desde la perspectiva de género, desde la crítica a la economía ortodoxa, pueden encontrarse distintos estudios y análisis que lo abordan, empleando para ello metodologías de diversas naturalezas; desde los que se limitan a hacer revisiones literarias hasta aquellos que realizan estimaciones econométricas para tener una evidencia empírica. Como ejemplo del primer caso, Quesada (2018), analiza la crisis económica y financiera desde la perspectiva de la economía feminista, crisis que los países no han podido superar completamente.

El trabajo de Quesada (2018) considera como centro de discusión la tendencia a masculinizar el estudio (específicamente) de la ciencia económica, puesto que los sustentos de ésta han dejado de lado el papel de la mujer en el desarrollo económico de las naciones a través de los años. Esta situación ha generado una problemática para los estudios de género, y en general para las mujeres, ya que fundamenta los diseños de políticas públicas para superar pobreza e inequidades de género. La autora contrapone las principales ideas de dos visiones: la economía feminista de la ruptura y la economía feminista de la conciliación, lo cual aporta 
un marco teórico para el artículo. La conclusión a la que llega es que existen, a la par de la económica, distintas crisis que tienen impacto directamente en las condiciones de vida de las mujeres, y que son naturales en el sistema capitalista, y mayor aún, que han sido desencadenadas por las políticas neoliberales implementadas por los gobiernos. Quesada propone la búsqueda de un nuevo modelo de producción y acumulación de renta y riqueza, ya que los modelos actuales han profundizado las desigualdades (sobre todo las de género).

Las aportaciones de Quesada encuentran una correspondencia muy notable en el modelo teórico propuesto por Noriega (2010). La premisa de la cual parte el autor para la construcción de su teoría es que, a diferencia de la teoría neoclásica, para quien existen sólo dos agentes representativos de la economía (consumidor y productor), deben incluirse elementos propios de la teoría del género, debido a que existen diferencias propias de ella que hacen que el comportamiento del agente sea distinto en caso de ser hombre y en caso de ser mujer. En este sentido, Noriega propone la existencia de dos consumidores: un hombre y una mujer madre. El artículo comprende que para una mujer madre, sus prioridades, dotaciones y utilidades son distintas, debido a que no solamente demandan producto para ellas, sino que también lo hacen para sus hijos. Además, el tiempo biológicamente disponible que poseen, no sólo lo destinarán al cuidado de sus hijos, sino también a la actividad productiva que les permita obtener el ingreso salarial con el cual consumirán producto para ellas y sus hijos (en caso de no contar con ingresos no salariales, como las ganancias de una empresa), mismo que habrán de distribuir según su criterio entre el número de hijos que tengan. La conclusión a la que llega el trabajo de Noriega (2010) es que la división sexual del trabajo, es decir, la distinción realizada entre hombres y mujeres por las actividades a las que se dedican según su género tiene implicaciones importantes sobre las asimetrías en ingresos (sobre

${ }^{5}$ Esta categorización refiere al concepto multidimensional de la pobreza, enfoque aplicado por el Consejo Nacional de Evaluación de la Política de Desarrollo Social (2017), donde el ingreso constituye la variable que mide todo salariales) entre dichos agentes. La economía de género debe estudiar, ante la realidad de una diferencia salarial entre hombres y mujeres, las posibilidades que tiene de reivindicar la equidad.

\subsection{POBREZA Y DISTRIBUCIÓN DEL INGRESO LABORAL: SER MUJER POBRE EN MÉXICO}

Hasta el año 2016, el Consejo Nacional de Evaluación de la Política de Desarrollo Social reportó que el número de personas en situación de pobreza (moderada y extrema) llegó a 53.4 millones de personas, equivalente (según la medición del CONEVAL) al $43.6 \%$ de la población. Es un problema que no ha sido eficientemente atendido si se considera que la pobreza ha agravado dado que en el 2008 la cifra no llegaba a los 50 millones de personas pobres (Consejo Nacional de Evaluación de la Política de Desarrollo Social, 2017). Boltvinik (2015) han señalado la importancia de atender las problemáticas que genera la pobreza: posterior a la crisis de 2009, el ingreso de los hogares mexicanos cayó en promedio $12.3 \%$, lo cual, aunado al aumento en el número de hogares, propició un incremento notable en el número de personas pobres y pobres extremas como porcentaje de la población total.

Boltvinik (2015) también señala que, la forma en la que se diseñan las políticas públicas cuyo objetivo es la disminución de la pobreza, depende en gran medida de la forma de medición de esta y esa medición, a su vez, depende de la manera en que es conceptualizada la pobreza. El problema se agrava aún más cuando se desagrega a la población pobre en hombres y mujeres, pues las estadísticas muestran que la cifra es mayor para la población femenina que para los hombres. Según datos del Instituto Nacional de las Mujeres (2016), de la población femenina en México hasta el 2016, solamente el $22.86 \%$ son consideradas no pobres y no vulnerables ${ }^{5}$. Si bien la pobreza es un problema global y que tiene afectaciones tanto en ámbitos urbanos como rurales; en personas jóvenes y

el bienestar económico, pero la vulnerabilidad se mide por la cantidad de derechos sociales a los cuales tiene acceso la población, o bien, el número de carencias que se tienen. 
mayores; en hombres y mujeres, también es cierto que no afecta en la misma medida a todos los grupos sociales.

Los Objetivos de Desarrollo Sustentable de la ONU (Comisión para América Latina y el Caribe, 2018), establecen la importancia de que las sociedades caminen hacia lograr la igualdad de género. La igualdad entre los géneros no es solo un derecho humano fundamental, sino la base necesaria para conseguir un mundo pacífico, próspero y sostenible. En ese sentido, un tema que recientemente ha cobrado importancia es el análisis de la pobreza (y específicamente de la pobreza laboral) desde la perspectiva de género. Desde el terreno de lo económico, el análisis de la discriminación hacia las mujeres ha derivado en problemáticas que constituyen el tema de investigación de diferentes estudios, tales como la concepción teórica de las bases de la propia ciencia económica (Quesada, 2018 y Noriega, 2010), la precarización laboral del trabajo femenino (Camarena, Saavedra, \& Ducloux-Saldívar, 2015), las limitaciones que enfrentan las mujeres al incorporarse a la fuerza productiva en la economía mexicana (Arceo \& Campos, 2014), las brechas salariales por género (Bidegain, 2016), entre otros.

Es la Economía Feminista (Rodríguez, 2015), la encargada de sentar las bases conceptuales para estudiar los problemas socioeconómicos de la mujer. Los resultados de dichos estudios indican que la pobreza ataca con mayor intensidad en el caso de las mujeres: el Instituto Nacional de las Mujeres reconoce que, para el caso mexicano en el 2016, había un mayor número de mujeres que de hombres en condiciones de pobreza y pobreza extrema (Instituto Nacional de las Mujeres, 2016). Al respecto, se muestra un indicador que ha construido la Comisión Económica para América Latina y el Caribe: el Índice de feminidad de la pobreza $^{6}$, el cual relaciona la tasa de mujeres en condiciones de pobreza con la tasa de hombres en condiciones de pobreza. Es importante resaltar que la

${ }^{6}$ La propia CEPAL indica: Si el valor del indicador es superior a 100 , significa que la pobreza afecta en mayor grado a las mujeres que a los hombres. Si el índice es menor que 100, la situación contraria. El índice de propuesta de este trabajo no afirma que para lograr la equidad de género los hombres y las mujeres deberían ser igualmente pobres, sino que las personas deberían poder superar la pobreza en igualdad de condiciones, sin importar si son hombres o mujeres.

Los resultados de la estimación del indicador de la CEPAL (2018) muestran que para el caso mexicano no necesariamente es así: medido desde el año 1989, la tasa de feminidad de la pobreza ha superado el $100 \%$, lo que implica que la relación es mayor a 1 y, por tanto, que la pobreza ha afectado más en el caso de las mujeres (CEPAL, 2018). Otra visión del problema se proporciona en el Gráfico 2, en donde puede notarse las proporciones de hombres y mujeres con respecto al total de la población en condiciones de pobreza. A pesar de que las cifras no son tan distantes una de otra $(25,541,041$ de hombres contra 27,877,110 de mujeres) lo realmente interesante es estudiar las causas y las implicaciones de que ello se presente así.

\section{Gráfico 1. Índice de Feminidad de la Pobreza. México,} 1989-2014

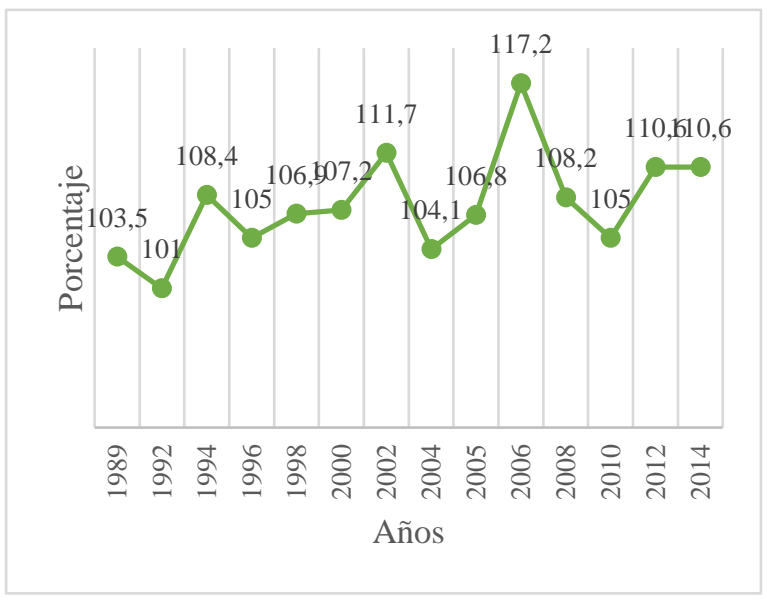

Fuente: Elaboración propia con base en los datos obtenidos de CEPALSTAT feminidad de la pobreza equivale a la relación entre la tasa de pobreza de las mujeres y la tasa de pobreza de los hombres. 
Gráfico 2. Población en situación de pobreza, distribución por sexo. México, 2016

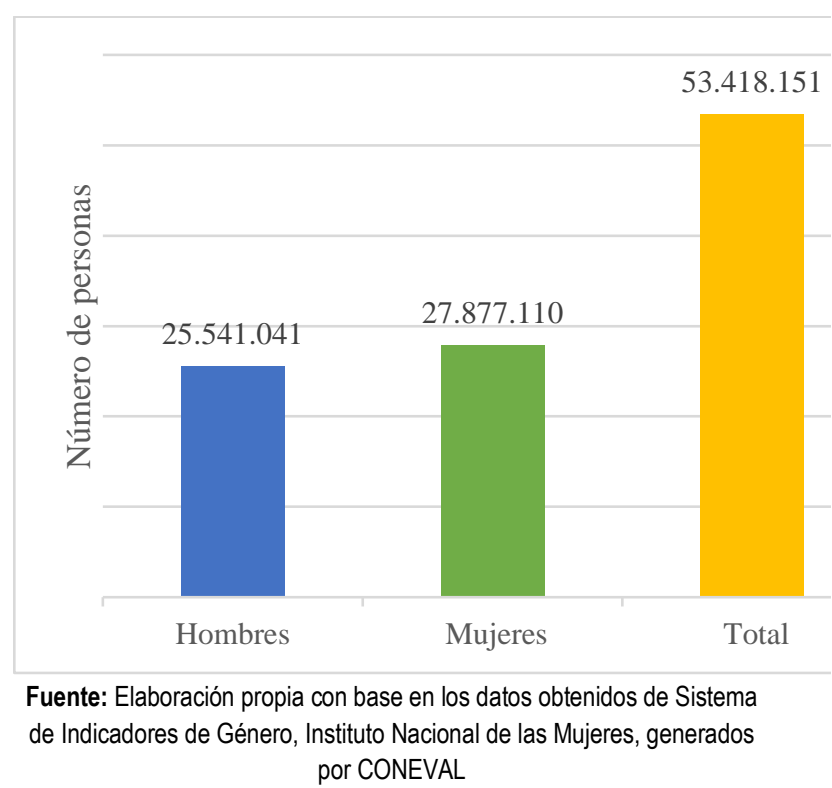

Las propias mediciones del CONEVAL permiten observar que existe un problema que se agudiza cuando se hacen diferenciaciones por sexo para el estudio, es decir, la pobreza es un problema que afecta gravemente a las sociedades, pero se agrava cuando se logra visualizar que existen diferencias importantes entre hombres y mujeres. Como puede verse en el Gráfico 3, en el primer trimestre del 2005 había un $20.5 \%$ de población ocupada femenina que no era capaz de adquirir la canasta básica alimentaria mexicana, contra un $13.5 \%$ de población ocupada masculina con la misma condición. Hacia el cuarto trimestre de 2018, las cifras se convirtieron en $19 \%$ y $12.1 \%$ respectivamente, es decir, en ambos casos disminuyó el porcentaje de personas ocupadas que no pueden adquirir una cesta alimentaria. ¿Esto significa una disminución en la brecha de pobreza laboral por género? ¿Puede afirmarse que existe un avance en el terreno de la equidad de género? Las respuestas son analizadas más adelante en este mismo apartado.
Gráfico 3. Porcentaje de la población ocupada con ingreso laboral inferior al costo de la canasta alimentaria según sexo, trimestral, 2005-2018

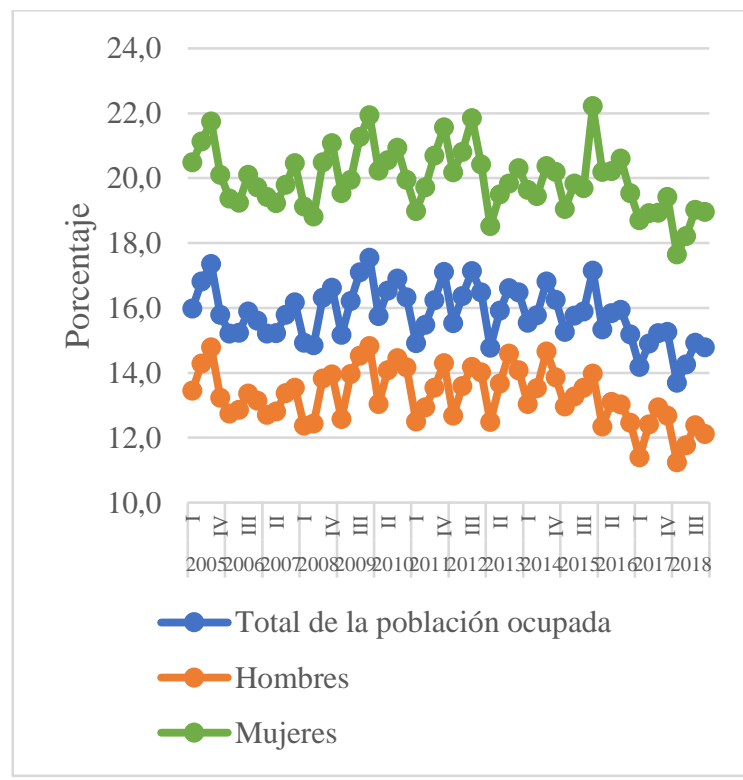

Fuente: Elaboración propia con base en datos de CONEVAL

Si bien hasta el momento el análisis ha permitido visualizar hasta cierto punto la realidad en la cual viven las mujeres pobres en México, para el Consejo Nacional de Evaluación de la Política de Desarrollo Social (CONEVAL), variables como el precio de los productos también intervienen en la medición y determinación de la pobreza, en tanto aumenta 0 disminuye el poder adquisitivo del ingreso de las personas. En este sentido, otra evidencia estadística importante es la que proporciona el mismo CONEVAL mediante la construcción del Índice de Tendencia Laboral de la Pobreza (ITLP), con el cual mide de manera trimestral la tendencia de la proporción de personas cuyo ingreso laboral no les permite adquirir una cesta básica alimentaria. Como lo indica el Consejo, el ITLP permite observar la evolución del poder adquisitivo del ingreso laboral de los hogares $y$, con base en éste, analizar si aumenta o disminuye el porcentaje de la población cuyos ingresos laborales son insuficientes para adquirir la canasta alimentaria. De esta manera, cuando el ingreso laboral es superior 
al valor de la cesta alimentaria, mejora el poder adquisitivo que tiene el ingreso laboral de las familias, por lo que el ITLP disminuye. Por el contrario, si los productos de la canasta alimentaria tienen un valor que excede al ingreso laboral, el poder adquisitivo disminuye, aumentando así al ITLP?

En el Gráfico 4, se construye una estimación del promedio simple del Índice de Tendencia Laboral de la Pobreza por año, para facilitar el análisis. Como puede observarse, para los primeros años de la estimación el ITLP es mayor para el caso de los hombres, situándose incluso por encima del promedio de la población ocupada en México. A simple vista, pudiera parecer que este indicador muestra lo contrario a la hipótesis de este trabajo: el ITLP es mayor en el caso de los hombres. Sin embargo, hay algunas cosas a debatir en este punto. En primer lugar, el propio CONEVAL considera que el ITLP no puede considerarse como una medida de pobreza, ya que no considera todas las dimensiones y conceptos que la medición formal de la pobreza toma en cuenta ${ }^{8}$. En este sentido, el ITLP no es evidencia suficiente para analizar la pobreza, pero sí aporta un panorama general sobre el poder adquisitivo del ingreso laboral.

En segundo lugar, la construcción de este índice considera el número de integrantes de los hogares, el cual según estimaciones al año 2015 del INEGI ascendió a un promedio de 3.9 personas en el caso de las familias con jefatura masculina y a 3.4 personas en hogares donde la jefa de familia es una mujer. Como puede notarse, las familias con jefe (hombre) de familia tienden a ser ligeramente más grandes que aquellas donde el mismo rol lo desempeña una mujer. Parecería que el hecho de que las familias con jefatura masculina tiendan a ser más grandes podría provocar que el poder adquisitivo de los hombres sea menor que el de las mujeres, dado que su ingreso laboral ha de dividirse entre más miembros tenga su familia. En otras palabras, podría esperarse que, si las familias con jefatura masculina son más numerosas, el ingreso por

${ }^{7}$ Si bien el Consejo manifiesta que no debe considerarse al ITLP como una medida de pobreza, para efectos del presente documento es útil porque integrante para tales familias sea menor que el de las familias con jefatura femenina. Sin embargo, esto no necesariamente ocurre así, dado que el ingreso promedio de los hombres tiende a ser superior al de las mujeres. Para analizar esto, es importante observar el ingreso laboral promedio por sexo en México.

observar el ingreso laboral promedio por sexo en México.

\section{Gráfico 4. Índice de Tendencia de la Pobreza Laboral en población ocupada según sexo, promedio simple anual, 2005-2018}

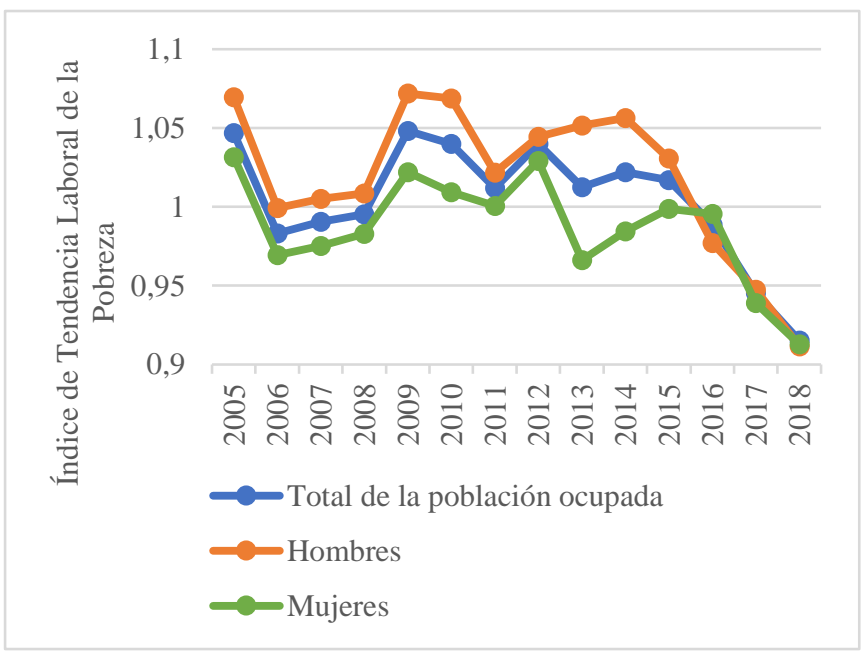

Fuente: Elaboración propia con base en los datos de CONEVAL

Con base en los datos proporcionados por el CONEVAL, puede observarse en el Gráfico 5 los ingresos laborales, tanto de hombres, mujeres, y total de la población ocupada, así como su evolución por trimestre desde el año 2005 y hasta 2018. El análisis estadístico indica que, en promedio para la población nacional, el ingreso laboral cayó de 4,568 pesos en el primer trimestre de 2005, a poco menos de 4,040 pesos en el cuarto trimestre de 2018. En el caso de la

permite visualizar el comportamiento estadístico de la cantidad de personas en condiciones de pobreza laboral, diferenciándolas por su sexo.

${ }^{8}$ Con base en la Ley General de Desarrollo Social vigente en México. 
desagregación por sexo, el ingreso laboral pasó de ser 5,076 a 4,410 pesos para los hombres, y de 3,662 a 3,457 pesos para las mujeres, durante el período antes mencionado.

Con esta información pueden obtenerse al menos dos conclusiones importantes: en primera instancia, que el ingreso laboral, sin importar el sexo del trabajador, en promedio ha tenido una tendencia a la baja del 2005 a la fecha. Esto significa que, de manera general, la tendencia en México es al empobrecimiento de los trabajadores, fenómeno que es observable tanto para hombres como para mujeres. En segunda instancia, puede asumirse que es cierto que la brecha salarial por género se ha ido acortando hacia los últimos períodos de estudio, esto es, la diferencia entre los ingresos laborales de hombres y mujeres es cada vez menor. Sin embargo, como puede notarse, las mujeres siguen en un nivel de ingreso laboral que casi no ha variado desde el 2005, de hecho, son incluso más pobres en el 2018 de lo que lo fueron en el 2005 porque su ingreso ha caído.

Aún más notable es el hecho de que el ingreso de los hombres ha disminuido en una cuantía visiblemente menor a la que ha tenido el ingreso laboral femenino: si se considera al año 2005 como el 100\% (un ingreso de 5,076 MXN), puede decirse que hasta el 2018, el ingreso de los hombres ha caído alrededor de $13 \%$. Explicado en otras palabras: la brecha de género en los ingresos laborales ha tendido a disminuirse, pero esto se debe a una disminución en los ingresos laborales de los hombres, y no a un avance en la equidad salarial entre hombres y mujeres. Esta conclusión resulta por demás interesante dado que contrasta en forma notoria con lo que se ha planteado desde el inicio del presente trabajo, no es que para lograr la equidad entre los sexos sea necesario empobrecer a los hombres, sino que hombres y mujeres se encuentren en igualdad de condiciones y oportunidades para superar la pobreza. La evidencia muestra que en la realidad medible y observable está ocurriendo completamente lo contrario a tal idea.

\section{Gráfico 5. Ingreso laboral promedio de la población ocupada a pesos constantes (real) según sexo, trimestral, 2005-2018}

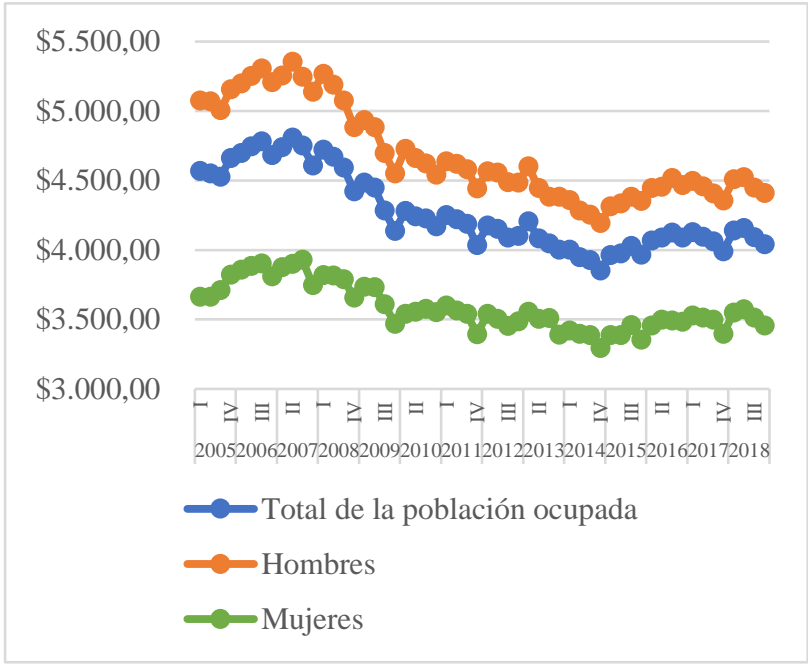

Fuente: Elaboración propia con base en los datos de CONEVAL

Lejos de creerse que la equidad implicaría que haya un número igual de hombres que de mujeres en condiciones de pobreza, o que hay que incrementar a los hombres pobres para que no haya diferencias por género en cuestiones de pobreza, el hecho de que la tasa de feminidad de la pobreza tienda a incrementarse (Bidegain, 2016), da la pauta para pensar que las características de la pobreza en mujeres son considerablemente distintas de las características en los hombres y, por lo tanto, que las causas que agudizan la pobreza en mujeres y hombres son diferentes.

\section{CONCLUSIONES:}

La evidencia empírica para el caso de México muestra que las mujeres son visiblemente más pobres que los hombres: el Consejo Nacional de Evaluación de la Política de Desarrollo Social muestra en la construcción de sus indicadores estadísticos que, considerando el total de personas que se encuentran en condiciones de pobreza, la mayor parte son mujeres. Si bien esta conclusión puede vincularse al hecho de que existe una composición mayoritariamente femenina de la población mexicana, también es cierto que, del total de mujeres, únicamente 
alrededor de $23 \%$ son consideradas no pobres y no vulnerables. Este fenómeno es más visible cuando se observan los niveles de ingreso laboral: considerando desde el primer trimestre de 2005 y hasta el último de 2018 puede observarse que la tendencia de los ingresos laborales de hombres y mujeres han tendido a disminuir. De primera vista, pudiera parecer que la brecha entre los ingresos laborales masculino y femenino se está cerrando, pero esto no es así debido a una tendencia a la equidad de género, sino a que el ingreso de los trabajadores ha caído en forma importante: lejos de que exista mayor equidad entre hombres y mujeres, la brecha comienza a cerrarse porque los hombres tienen un ingreso laboral cada vez menor, mientras que las mujeres siguen siendo prácticamente igual de pobres en 2018 de lo que lo eran en 2005 (incluso, un poco más). La realidad en México es poco alentadora para las mujeres trabajadoras: lejos de procurarse que las retribuciones sean equitativas ante actividades iguales, ellas cada vez se empobrecen un poco más, mientras que sus compañeros hombres también tienden a empobrecerse, aunque ciertamente, siguen percibiendo más que ellas.

Es muy interesante observar las distintas perspectivas teóricas y empíricas que buscan explicar e interpretar el fenómeno: si bien la teoría ortodoxa encamina su interpretación de la pobreza como un fenómeno ajeno al funcionamiento de los mercados, existe toda una crítica construida alrededor de la hipótesis que plantea la existencia de una sociedad simétrica que no da paso a los problemas de distribución del ingreso. Aunque pudiera parecer en inicio que los trabajos que argumentan la crítica contra la economía dominante, en realidad la mayoría de ellos encuentra correspondencia en los postulados de la economía clásica, según la cual existen asimetrías en la sociedad, dando paso a la conformación de clases sociales y, por lo tanto, a un conflicto distributivo.

En suma, existen dos problemáticas: por una parte, el conflicto distributivo, puesto que existen asimetrías visibles en la distribución del ingreso, la cual es benéfica para el capitalista. Por otra parte, dentro de la porción poblacional que acumula menor cuantía del ingreso, esto es, dentro de la clase trabajadora, existen asimetrías que vulneran las condiciones de vida de las mujeres trabajadoras, lo cual va en contra de la equidad de género y del empoderamiento femenino.

\section{REFERENCIAS:}

Andreozzi, L. (2018). Pobreza por Ingreso y Tiempo en la Ciudad de Rosario. Astrolabio Nueva Época, 213-232.

Arceo, E., \& Campos, R. (2014). Evolución de la Brecha Salarial por Género en México. El Trimestre Económico (VII), 619-653.

Arriagada, I. (2005). Dimensiones de la pobreza y políticas desde una perspectiva de género. Revista de la CEPAL, 101-113.

Benería, L. (2006). Trabajo Productivo / Reproductivo, Pobreza y Políticas de Conciliación. Nómadas, 8-21.

Bidegain, N. (2016). Desigualdades de género y brechas estructurales en América Latina. Nueva Sociedad, 50-57.

Boltvinik, J. (2015). Dos metodologías y dos visiones de la evolución de la pobreza en México, 2008-2010. Rúbricas, 29-35.

Boltvinik, J., \& Hernández Laos, E. (2001). Pobreza y Distribución del Ingreso en México. México: Siglo XXI.

Camarena, M., Saavedra, M., \& Ducloux-Saldívar, D. (2015). Panorama del género en México: Situación actual. Revista Científica Guillermo de Ockham, 77-87.

Comisión Económica para América Latina y el Caribe. (2018). Estadísticas e Indicadores Sociales. Obtenido

de http://interwp.cepal.org/sisgen/SisGen_Muest raFicha. asp?indicador=1694\&id_estudio=362

Comisión para América Latina y el Caribe. (2018). Agenda 2030 y los Objetivos de Desarrollo Sostenible. Una oportunidad para América Latina y el Caribe. Santiago: Publicación de las Naciones Unidas.

Consejo Nacional de Evaluación de la Política de Desarrollo Social . (2017). Medición de la pobreza en México y en las Entidades Federativas 2016. México: CONEVAL. 
Consejo Nacional de Evaluación de la Política de Desarrollo Social. (2016). CONEVAL. Recuperado el 10 de Octubre de 2018, de Medición de la pobreza: https://www.coneval.org.mx/Medicion/Pagina s/Pobrezalnicio.aspx

Consejo Nacional de Evaluación de la Política de Desarrollo Social. (2017). Medición de la Pobreza. Recuperado el 08 de Septiembre de 2018, de Pobreza en México: https://www.coneval.org.mx/Medicion/Pagina s/Pobrezalnicio.aspx

Consejo Nacional de Evaluación de la Política de Desarrollo Social. (s.f.). Medición de la Pobreza. Recuperado el 05 de Abril de 2019, de Índice de la Tendencia Laboral de la Pobreza:

https://www.coneval.org.mx/Medicion/Pagina s/Indice-de-la-tendencia-laboral-de-lapobreza-(ITLP).aspx

Cruz, J. (2013). La distribución del ingreso y los modelos de desarrollo en México. México: UNAM, Instituto de Investigaciones Económicas.

Esquivel, G. (2015). Desigualdad extrema en México: Concentración del poder económico y político. México: OXFAM.

Instituto Nacional de Estadística y Geografía. (2015). Hogares y vivienda. Obtenido de Características de los hogares. Tamaño promedio de los hogares por entidad federativa según jefatura del hogar: http://www.beta.inegi.org.mx/temas/hogares/ default.html\#Tabulados

Instituto Nacional de Estadística y Geografía. (2015). Población. Obtenido de http://www.beta.inegi.org.mx/temas/estructur al

Instituto Nacional de las Mujeres. (2016). Sistema de Indicadores de Género. Obtenido de Pobreza: http://estadistica.inmujeres.gob.mx/formas/pa norama_general.php?menu1=10\&IDTema $=1$ 0\&pag $=1$

Klimovsky, E. (2000). Modelos Básicos de las Teorías de los Precios. Cuadernos de Economía, 77103.
Marx, K. (1867). El Capital. Tomo I. México: Fondo de Cultura Económica.

Moctezuma, D., Narro, J., \& Orozco, L. (2014). La mujer en México: Inequidad, pobreza y violencia. Revista Mexicana de Ciencias Políticas y Sociales, 117-146.

Noriega, F. (2010). Microfundamentos para la Economía de la Mujer. Economía y Sociedad, XIV(25), 69-89.

Oaxaca, R. (1973). Male-Female Wage Differentials in Urban Labor Markets. International Economic Review, 14(43), 693-709.

Obregón, C. (2008). Globalizacion y Subdesarrollo. México: Pensamiento Universitario lberoamericano.

Ochman, M. (2016). Políticas sociales y empoderamiento de las mujeres. Una promesa incumplida. Estudios Políticos, 3251.

Ordóñez, G. (2018). Discriminación, pobreza y vulnerabilidad: los entresijos de la desigualdad social en México. Región y Sociedad, 1-30.

Organización de las Naciones Unidas. (2018). Informe de los Objetivos de Desarrollo Sostenible. Nueva York: Naciones Unidas.

Pedrero, M., Rendón, T., \& Barrón, A. (1997). Segregación ocupacional por género en México. Ciudad de México: Universidad Nacional Autónoma de México.

Peña, X., \& Uribe, C. (2013). Economía del Cuidado: Valoración y Visibilización del Trabajo No Remunerado. Lima: Instituto de Estudios Peruano.

Piketty, T. (2014). El Capital en el Siglo XXI. México: Fondo de Cultura Económica.

Quesada, R. (2018). Dimensiones de la Economía Feminista: Claves para una redefinición. Cuestiones de género: de la igualdad y la diferencia, 47-66.

Ricardo, D. (1817). Principios de Economía Política y Tributación. España: Sarpe.

Ricossa, S. (2002). Diccionario de Economía. México: Siglo XXI.

Rodríguez, C. (2015). Economía feminista y Economía del cuidado. Aportes conceptuales para el estudio de la desigualdad. Nueva Sociedad, 30-44. 
Sen, A. (1981). Poverty and Famines: An Essay on Entitlement and Deprivation. New York: Oxford University Press.

Smith, A. (1776). Investigación sobre la naturaleza y causas de la riqueza de las naciones. México: Fondo de Cultura Económica.

Stiglitz, J. (2012). El Precio de la Desigualdad. España: Taurus.

Velázquez, D., Rodríguez, E., \& González, J. (2017). Conflicto distributivo entre salarios y ganancias: Revisión teórica. Pachuca: Universidad Autónoma del Estado de Hidalgo. 